\title{
DEVELOPMENT AND IMPLEMENTATION OF MOBILE GEAR For SKIER TRAINING SYSTEM
}

\author{
Yong-Wook $\mathrm{Kim}^{1}$ and Sung-Rak $\mathrm{Choi}^{2}$ \\ ${ }^{1,2}$ Department of Sport Science, Hanyang University, Korea
}

\begin{abstract}
The purpose of this study is to develop a Skier Training System for monitoring skiing courses and analyzing skier posture using smartphones. The system is composed of three elements: first, operating servers that receive information on the skier's exercise; second, Skier Training Apps developed using Java and C based on Android SDK 4.2 or above; and third, Data Gathering Gear embedded with gyroscope sensors and GPS for measuring skier stance. When a skier wearing our Data Gathering Gear runs down a slope, his or her stance is uploaded to the operating servers. Then the servers calculate the recorded data, such as velocity and turn trajectory, in order to suggest body angles that are optimized for the skier; subsequently, the skier can improve his or her skills by checking his or her records, and then attempt to correct his or stances based on the suggestions offered by the Skier Training Apps.
\end{abstract}

\section{KEYWORDS}

Skier Training, Mobile Apps, Mobile Gear

\section{INTRODUCTION}

Many methods have been applied to improve the sports ability of athletes. Sports biomechanics, physiology, and psychology are used to directly measure and evaluate physical ability, and thus suggest new training methods. Such training methods were employed even before computers were invented. Since the development of computers and their practical uses in daily life, many studies have been performed, particularly in the sports training sector. The computer has been used to find the most effective methods for accurately measuring and improving the exercise ability of elite athletes. As computers develop, various scientific sports programs are being developed to measure and record the physical ability of athletes and manage their conditions. Recently, the current research environment has been expanded along with the emergence of smartphones. In the past, several sensors were attached to an athlete's body, and then the sensors needed to be connected to a computer by wire in order to measure, in detail, the exercise records of elite athletes. Such devices, however, posed some issues, such as uncomfortable or unnatural movement of athletes and network disconnection while recording.

Today, smartphones can replace the computer. Smartphones use mobile communication networks to connect to other computers anytime and everywhere. In addition, GPS, gyroscope, acceleration, illuminance, and other sensors embedded in smartphones can collect various types of information [1]. Therefore, the collection of smartphone-based information can be performed free from location limits. In addition, wired smartphone connections can be resolved through various sensors in the Bluetooth wireless module. Along with the development of various equipment, including smartphones, many developments and studies are ongoing for improving the athletic skills of elite athletes [2]. Studies that require massive human resources and investment in order to improve the athletic skills of elite athletes are important. However, additional studies on sports training applications (apps) are also required to boost simple and convenient uses in ordinary people. Studies on mobile apps that can help improve user lifestyles

DOI : 10.5121/ijsea.2017.8102 
International Journal of Software Engineering \& Applications (IJSEA), Vol.8, No.1, January 2017

are also performed in the medical sector. Various apps have been developed for the purpose of treating or adjusting patients, such as mobile apps for correcting patients' incorrect posture or inducing an active lifestyle in teenagers $[3,5]$. If users employ the aforementioned mobile apps well, they can realize their treatments or adjustments [6,7]. Smartphones are considered a convenient device for users because the latter can install and employ various apps in their smartphones. In other words, users can employ various apps simply by downloading them from a mobile store and installing them into their smartphones [8].

If users have a strong will for training, sports training apps can help them achieve their desired training performance without temporal or location limitations. Through these apps, users can control their skills and check their exercise logs to obtain feedback. Typically, athletes have had to check training logs monitored by a third person, but app users can improve their skills through self-reports (self-feedback) [9]. If skiers have access to a monitoring tool that allows them to check their downhill records, such tool could help the skiers improve their skills. Therefore, the purpose of this study is to design mobile apps required for skiers and explore the applicability and effectiveness of mobile apps at ski slopes. This study aims to design convenient mobile apps for skiers. The mobile apps designed in this study include a monitoring feature for skier exercise logs and postures. Furthermore, user-friendliness with easy-to-use features is important, not to mention mobility and 24-hour usability.

\section{RESEARCH APPROACH}

Using smartphones to develop a training system entails a few restrictions. For example, it is difficult for athletes to collect exercise data on their own, and therefore, they require assistance from people responsible for measuring and recording the data. This is the reason a sensor for measuring and logging athlete movements is necessary, and mobile apps that can input such data are required. In short, a sensor for collecting information and mobile apps for entering the information are required. First, let us look into mobile apps that have been widely used in many sectors, which are largely divided into three: health and fitness, exercise tracking apps, and motion tracking apps.

The mobile apps currently available in the health and fitness sector vary from providing instructions on how to perform a certain behavior and modeling/demonstrating the behavior, to providing feedback on performance [10]. The sector is further classified into three broad types of training apps (health, exercise, and motion), which are briefly described in the following paragraphs.

First, the key functions of health training apps include the following: the users exercise with their own posture as guided by a personal trainer in a video (e.g., [11-13]). First, the trainees learn about exercising for health training. Next, the trainees determine the exercise type based on their purpose for exercising (weight loss, muscular strength, etc.). Based on the exercise type determined by the trainees, the exercise program, as recommended by the health training app, is executed. Exercise logs are saved in the trainees' smartphones for the users to review. However, such exercise programs pose the following problems. Users exercise without measuring individual exercise intensity. Health trainers at fitness clubs draw exercise programs that consider different trainee variables. All items from the measurement of basic exercise unit, '1RM', intensity from exercising to muscular training should be accurately determined. However, health training apps require users to enter all variables, thus allowing the possibility of entering incorrect variables. This further poses limits for creating accurate exercise programs suitable to each individual. This problem associated with the variables that should be directly entered by users is also found in the mobile apps of other sectors. Health training apps, which should suggest an exercise prescription without accurately checking the personal characteristics of users, pose 
limitations for general exercise prescription. Despite these limitations, this program continues to grow and develop for improving user convenience [14]. Therefore, several variables should be considered in developing health training apps, in addition to avoiding making incorrect exercise programs.

Second, exercise tracking apps are considered to be nearly complete. Apps are either already embedded in smartphones from their release, or sold at app stores. The similarity of these apps is that user movements are measured by GPS-based location traceability or gyroscope sensors embedded in smartphones or smart watches [15]. If users perform an outdoor exercise, such as running or biking, these apps allow tracking and recording the user's route and speed very accurately. In addition, the conditions of the outdoor environment, such as local weather information, are provided to give users various logs. Moreover, users can make their own exercise routes using the information shared by other users. People who actively use exercise tracking apps can easily post and share their individual exercise logs and lifestyles on social media.

Third, motion tracking apps adjust user position when users exercise by employing some equipment. Such motion tracking apps are already vitalized in the golf sector and are presently available in the market (e.g., [16]). A device that can be attached to a golfer's hat and serves as a guide for the distance from the hitting point to a hole on the golf field has been successfully commercialized [17]. In addition, there are other apps that analyze the user's swing posture in order to instruct the correct posture via a device attached to the golf club [18]. Other apps are being developed and sold in the tennis sector. Such apps require a device attached to the tennis racket in order to analyze the user's tennis swing, and they can propose an accurate hitting point (e.g., [19]). These products are operated by attaching a simple device to the sporting goods, which is then connected to smartphones by Bluetooth in order to trace and record the user's exercise motions. The major manufacturers of sporting goods, such as Nike and SONY, sell mobile apps designed for popular sports, such as golf and tennis, to support sports enthusiasts. Once such enthusiasts are satisfied with the mobile apps, customer satisfaction in the existing business area is improved [20]. The reason for many major global manufacturers of sporting goods to sell mobile apps and various accessories is to conserve consumer loyalty. Therefore, the market in sports mobile apps is expected to grow continuously.

Different types of sensors have been developed to collect athlete motions. One such sensor for checking athlete actions is based on RFID technology. Wearable sensors around athletes' joints

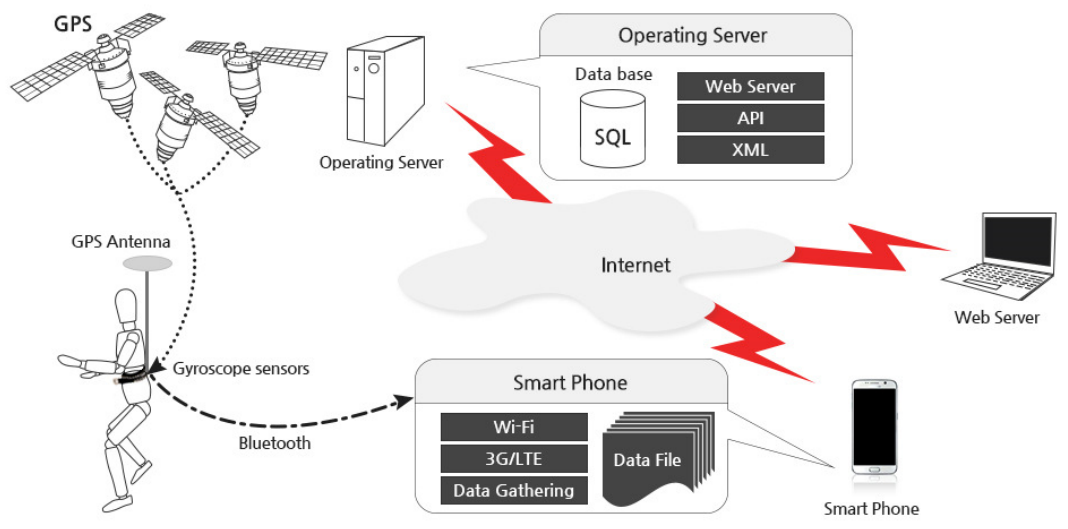

Fig. 1. Diagram for Skier Training System 
gather a variety of information. For instance, the National Hockey League (NHL) in North America installed such sensors within athlete equipment during the 2015/2016 season to provide real-time match information [21]. The National Basketball Association (NBA) offers a player tracking service that is based on video analysis technique. Spectators can obtain information on player performance in real time via smartphones or computers [22]. These services require data collection systems with enormous storage space. Because such systems are not available to ordinary users (to the best of our knowledge), this study intends to develop a skier training system capable of developing and operating a simple device that skiers can use to collect exercise data. Downhill skiing as a sport requires a basic downhill technique. Most ordinary skiers enjoy going downhill at ski resorts. Downhill skiing requires proper technique in order to safely ski down a slope: the skier should focus his or her body weight at an accurate center point. The body angle should be properly tilted toward the inner turning axis in order for skiers to stably spin at a fast speed. The body angle should be changed in proportion to speed and turning radius. Skiers learn the proper body angles through experience. However, if skiers could be advised on how to control their body angle, they might easily acquire advanced skills. In this study, the location of a skier collected via GPS is analyzed to check the skier's downhill records. Therefore, this study aims to assist in the design of skier training apps that propose proper body angles at specific locations. A skier's location is collected via GPS, and the skier's posture is recorded in the smartphone via gyroscope sensors. The information recorded in the smartphone is saved on an operation server in real time. In addition, the proper body angle at a particular location is simultaneously calculated and recorded by the operation server. Skiers can check their performance logs after skiing. The operation servers can then advise the skiers on their best body angle at each turning posture. By comparing their own postures and best body angles proposed by the operation servers, skiers can improve their skills.

\section{Proposed Methodology for Designing ANd Developing Mobile GEAR}

The purpose of this study is to develop a skier training system that collects and analyzes the skier's posture information in real time. Currently, the hardware embedded in smartphones is highly developed and comparable to that of personal computers. However, smartphones require and use many resources in order to perform the basic functions of a telephone, such as calling, messaging, etc. Thus, smartphones pose difficulties in multi-tasking. The skier training apps designed in this study collect information and transfer it onto an operating server as a primary role. Instead of performing the required calculations on the smartphones, the applications send the information to be calculated to online operating servers. In other words, required calculations, such as Recommend Angle Adjustment (RAA), are performed on an operating server. Therefore, smartphones simply upload exercise information gathered through gyroscope and GPS sensors onto the operating server in real time. In general, the operating server reports the outcomes of such information analysis.

\subsection{Skier Training System}

Fig. 1 shows a scheme for the proposed Skier Training System. This study is broadly designed with three subparts: 1) operating servers, 2) skier training apps operated on smartphones, and 3) data gathering gear. The information collected by the smartphones is uploaded onto the operating server in real time (Figure 1). If the information fails to upload because of poor network connection, it is temporarily saved in the smartphone and automatically uploaded once the network is back online. In the operating server, the database system is operated to manage user information. An identification number and user ID are both used as the user's identification information. When operating skier training apps in the smartphone, the apps continue to operate in a background process and automatically collect information. The proposed skier training app 
International Journal of Software Engineering \& Applications (IJSEA), Vol.8, No.1, January 2017

automatically performs its function immediately after the skier arrives at a ski resort. Presently, the proposed app stores GPS data gathered from ski resorts in Korea and Japan. However, the ski resort maps shared among various skier training apps are constantly updated with new information.

\subsection{Development Environment}

The development environment for this study is as follows: the operating server system is composed of database and communication systems. The database system is built based on Oracle Database 12c. The communication system for the skier training apps is made using XML. The skier training apps are developed using Android SDK 4.3, Java, and C-language. In addition, an IOS version of the apps has been planned for development in order to be available on iPhones.

\subsection{Information Gathering}

Fig. 2 shows the scheme for the sensors that collect skier exercise information. The skier training apps used with the skiers' smartphones are connected to two different sensors, both of which are responsible for collecting downhill ski records from a skier. The GPS sensor collects the data of the skier's current location. If three or more GPS signals are received, an accurate location within five meters of the skier's actual position can be collected. The collected GPS information is combined with the location data from the user's mobile phone network to provide a more accurate location for the skier. The other sensor is a gyroscope that checks skier posture. When the skier wears the gyroscope sensor belt and connects it with his or her smartphone via Bluetooth, the gyroscopes on the belt are activated and ready to use. When a skier skis downhill, the position is recorded in real time via the gyroscope sensors in the smartphone, and then uploaded to the operating server. The data collected from both the data gathering gear and smartphone are uploaded simultaneously. The operating server corrects the collected data.

\subsection{Mobile App Design}

Top priority for the design of the proposed mobile app was given to user-friendliness. The proposed skier training app was designed for automatic execution without requiring special operation. Such training app was also designed to be intuitive to the user. The skier completes all

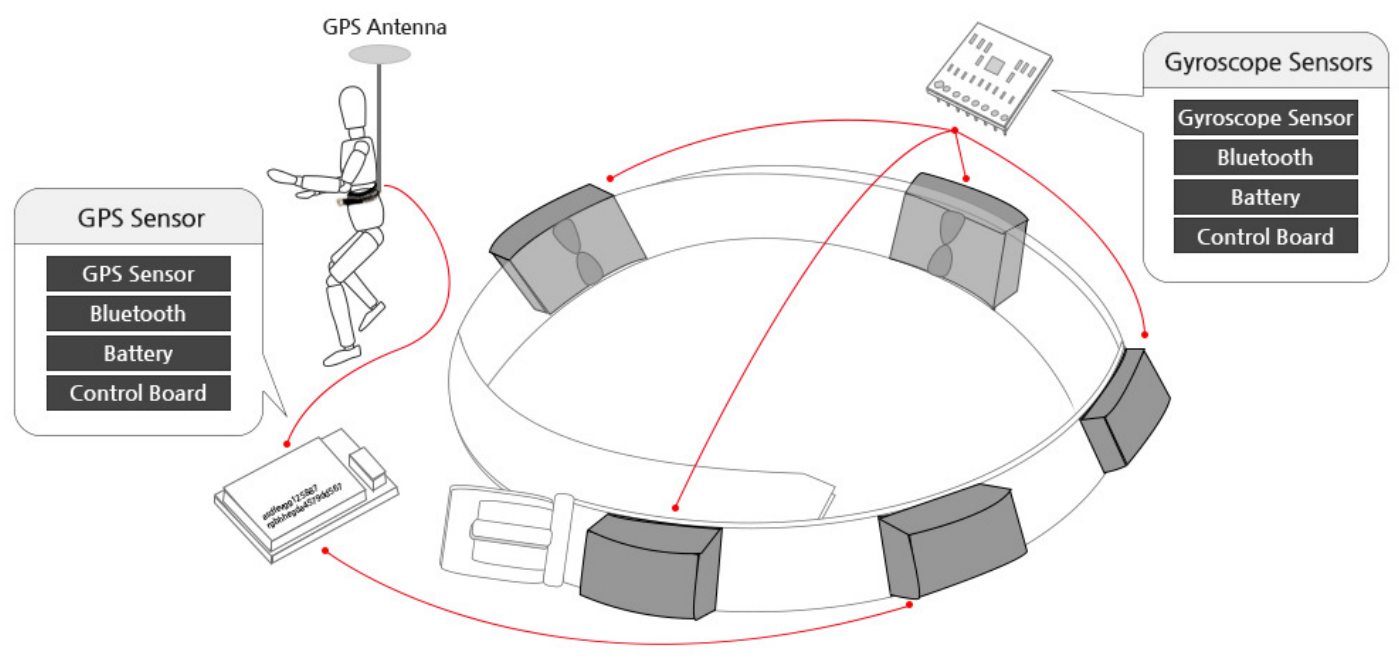

Fig. 2. Data Gathering Gear 
without requiring special operation. Such training app was also designed to be intuitive to the user. The skier completes all preparations by pressing the start button. A skier only needs to check the operation of our proposed skier training app once prior to skiing. Once the proposed app is activated, the skier is voice-guided accurately based on his or her travel distance and speed.

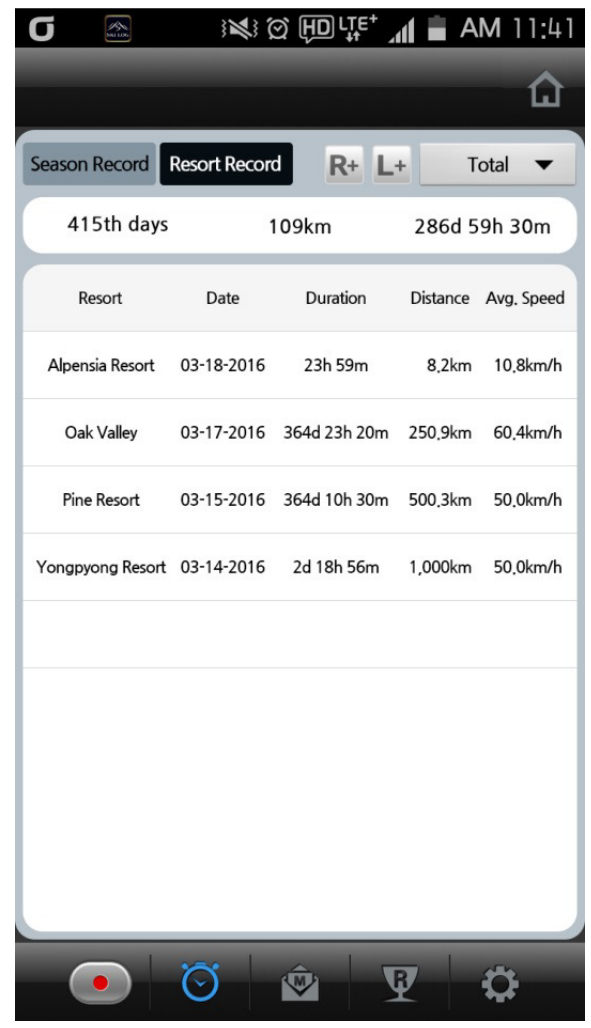

Fig. 3. Skier Training Record

If the skier training app is already running when the skier goes to the ski resort, the app works in the background of the smartphone's operation to determine whether the skier boards a ski lift. After confirming that the skier has boarded a ski lift, the skier's activity information is automatically saved on the operating server (Fig. 3). Even if the skier were to forget to turn off the app once he or she left the ski resort, the app would automatically turn off immediately after the skier left the resort, without requiring the user to press the stop button after skiing. Skiers can check their ski logs while resting. The log is recorded and listed by season, location, and date. Each log allows users to check their exercise routes, positions, etc. In smartphones, the low priority process can be exited without prior warning in order to ensure the necessary resources to execute an application program. For this reason, users of the proposed skier training app have voiced-guided logs that are activated immediately after they board a ski lift. The users can listen to real time log broadcasts and know the operation status of their skier training apps.

\section{FINDING AND DISCUSSION}

Field tests for the proposed skier training app were performed at six different ski resorts in Korea and two ski resorts in Japan during the 2015/2016 season. Seven different smartphone types, including the Samsung Galaxy S6, were used in this experiment. A total of six skiers participated in the test. The level of the GPS signals received from each resort was different. Regardless of 
this limitation, there were no problems with the accuracy of the skier's location. Disconnection points caused by weak GPS signals while skiing were also observed.

At those times when a skier could not be located by the GPS satellites, the operating server predicted and estimated the missing location information by calculating the last known position with the current one. In addition, the location information obtained via GPS data and mobile phone networks was adjusted and updated on the operating server (Fig. 4).

The standard time used for the calculations was not based on GPS time, but on the operating server's time regardless of time zone. The time shown in the ski log in the same as that on the user's smartphone. Skier speed was calculated by 3D coordinates through the skier's latitude, longitude, and altitude. The irregularity of the travel route was not considered. However, a deviation greater than $10 \mathrm{~m}$ was found in the altitude information received by the GPS; therefore, a Google elevation API was used to adjust the GPS altitude. Skier speed was calculated considering a $10 \mathrm{~s}$-skier distance as the basic unit. Skier speed was recorded every $0.5 \mathrm{~s}$ and 60 different linearized measurements were used to calculate the average speed once irregular speed errors were removed.

Posture information collected via the gyroscope sensors was measured and calculated more precisely than speed. Once changes in a skier's posture were detected by the gyroscope sensors, the sensor collected information every $0.1 \mathrm{~s}$. A total of 50 linearized postures was collected, and thus the skiers' posture-change data were more accurately calculated and recorded.

Skiers rarely check their smartphones while going downhill, and thus real-time RAAs are not calculated. However, such RAAs are calculated immediately after a skier uploads his or her location and posture data onto the operating server. Users can receive feedback when they check their records while resting after skiing (Fig. 5). RAA is calculated based on the body angle proposed in the research for downhill posture of alpine skiers [23, 24]. Aside from the recommended body angle of such research, the skier body angle was also calculated based on the speed and directional turning track data from our study participants.

The RAA proposed in this study was calculated by assuming that the stance of the skier's head, upper body, and lower body is obtained in a straight line. This is disparate from stances obtained while the skiers participate in downhill, mogul, or slalom racing. This is also different from the short turn usually employed in InterSki. Turning stances that are based on ski skills are significantly different from those in the proposed RAA. Therefore, RAA should be used simply as a reference for checking changes in the center of gravity. 
International Journal of Software Engineering \& Applications (IJSEA), Vol.8, No.1, January 2017

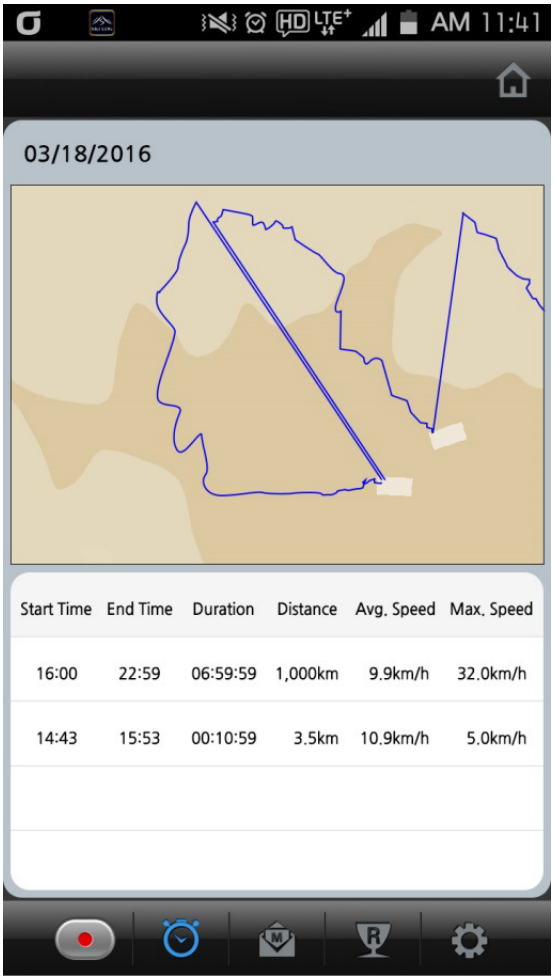

Fig. 4. Tracked Route

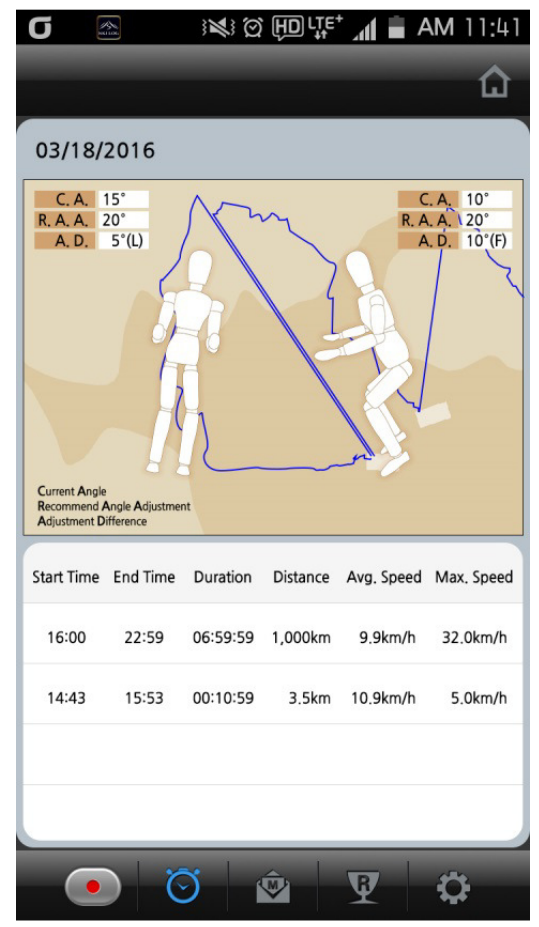

Fig. 5. Recommend Angle Adjustment 


\section{Conclusions}

\subsection{Conclusion}

Ski enthusiasts are not satisfied with their skiing skills. To be precise, they are dissatisfied with the inability of improving their skills. Although skiers are eager to develop such skills, not many can improve with master level training courses. Using the skier training app developed in this study, skiers can check their downhill trajectory, among other things. The skiers can also check their downhill position at a particular location. A skier's body angle changes based on the skier's speed and turning radius while skiing downhill. This information is collected and analyzed in order to recommend the best body angle for improvement. Consequently, the skier can make an effort to improve his or her downhill skiing position compared with the recommended body angle. The proposed mobile app allows skiers to check and adjust their posture in order to help them improve their skiing techniques. In addition, skiers can check their ski log data for information such as duration, distance, calories consumed, etc. This information can help skiers establish their own exercise plan during the winter season. Therefore, the skier training app developed through this study produced the results discussed in the following paragraphs.

First, skiers can check their ski log. The location collected via GPS is analyzed to record the skier's travel route. Next, detailed information, such as longitude, latitude, and altitude, are calculated for a skier's particular location in order to provide recommended average speed, maximum speed, turning radius, etc. Personal information entered previously, such as the skier's weight, height, age, etc., is used to estimate calorie consumption. The above information is gathered for skiers to set up an exercise plan.

Second, skiers can adjust and improve their posture. The most important measurements for downhill skiing postures are joint and body angles. In this study, we focused on body angle measurements. The information recorded from mogul ski and slalom sports that require instant quick movements from professionals was not consistent with this study, which analyzed the middle and long turns performed by ordinary skiers while predicting their downhill postures. Specific torso, hip joint, and knee angles were crucial factors for the downhill postures measured in this study. The skier training app developed in this study analyzes skier information (speed, turning radius) in order to recommend the proper body angle. However, the RAA proposed in this study resulted from a simple mechanical calculation that did not consider the skier's height, weight, and age. Our RAA is provided as reference information for the skier, but it is not essential for the skier to follow.

Third, exercise management programs were first used by elite athletes, and are now practiced by ordinary skiers. All sports enthusiasts want to improve their sports technique. Elite athletes are systematically and scientifically managed by a training system of professional coaches in order to improve their athletic ability and performance. However, ordinary skiers can employ exercise management program mobile apps to manage their skills.

Smartphone app technology information has been developed from the user's perspective, and then analyzed and applied. For example, mobile apps that allow dieters to check their food consumption and calories via voice instruction have been developed [25]. In addition, smart miniscreens attached inside skier goggles to provide information such as skiing time, distance, altitude, and more, are already available on the market (e.g., [26]). Applying this technology proposes additional studies, such as those described in the following paragraphs.

The combination of a camera that can be installed on a skier's goggles for analyzing the downhill route and a sensor attached to the skier's legs for measuring and recording joint angle information 
can produce data used in audio suggestions received by the skier while skiing. In addition, foot pressure sensors attached to the skier's boots could be used to propose more precise and stable postures. Such sensors could be integrated with wearable devices, such as chest sensor belts already available on the market that can measure heart rates [27]. As mentioned above, various types of physical information can be collected from skiers. Therefore, the best exercise program can be recommended using data collected for comprehensive calculation.

More studies are required on the development of various sensors that can collect skier exercise data, as well as on processing the collected information. All studies should be conducted with a user interface from the user perspective for easy and simple uses, and should be designed based on a strategy applicable to the sales market [28]. Most of the currently available mobile apps allow users to record their exercises and obtain feedback [29]. If users would check their training content and make an effort to improve their skills, mobile apps could help them [30-32]. If skiers choose to properly use the information proposed in this study, their techniques and exercise skills would improve.

\subsection{Recommendation and Research Limitation}

The goal for developing the proposed skier training app and data gathering gear is to commercialize them. However, there are several obstacles to commercialization. In terms of functional problems, first, the RAA suggested by the skier training app cannot be generalized. Second, it is not easy to maintain a stable connection between the data gathering gear and smartphones: for example, five sensor data items sent from the data gathering gear should have been uploaded in real time, but Bluetooth was frequently disconnected. Third, reducing antenna weight in order to accurately receive data from GPS devices is urgent. The measurement error for GPS data received on smartphones is 5 to $10 \mathrm{~cm}$. Therefore, a precise GPS receiver with a maximum of $1 \mathrm{~m}$ measurement error was installed in the data gathering gear. However, an antenna with a diameter of at least $20 \mathrm{~cm}$ is needed for accurate data reception, and it is next to impossible for a skier to enjoy skiing with an antenna on his or her body.

Nonetheless, all the functional issues can be solved technically. The most significant hindrance is financial. Raising unit prices is the only way of resolving the aforementioned functional problems. Unfortunately, the unit prices for the data gathering gear developed as a pilot product in this study indicate that the product cannot be commercialized. We required five Bluetooth modules, four gyroscope sensors, one GPS sensor, five control boards, and five batteries in order to make the proposed data gathering gear, and thus it is unfeasible to lower the product price regardless of how the unit prices are adjusted. However, an expensive product cannot be competitive in the market. In contrast, the proposed skier training app developed in this study did not incur any costs. The app can be used after a simple download and installation. Thus, developing skier training apps that can operate without installing data gathering gear is most practical. However, data such as RAA might not be supplied because of insufficient information collection.

\section{NoTE}

This study is an updated and extended paper of the proceedings that were presented at icSPORTS 2015'. 
International Journal of Software Engineering \& Applications (IJSEA), Vol.8, No.1, January 2017

\section{REFERENCES}

[1] Dobkin BH, (2013) "Wearable motion sensors to continuously measure real-world physical activities," Curr Opin Neurol, vol. 26, no. 6, , pp. 602-608.

[2] Chardonnens J, Favre J, Cuendet F, Gremion G, Aminian K , (2014) "Measurement of the dynamics in ski jumping using a wearable inertial sensor-based system,” J Sport Sci, vol. 32, no. 6, pp. 591600.

[3] Gefen R, Dunsky A, Hutzler Y, (2015) "Balance training using an iPhone application in people with familial dysautonomia: three case reports," Phys Ther, vol. 95, no. 3, pp. 80-388.

[4] Lubans DR, Smith JJ, Skinner G, Morgan PJ, (2014) "Development and implementation of a smartphones application to promote physical activity and reduce screen-time in adolescent boys," Frontiers In Public Health, vol. 2, no. 42, pp. 1-11.

[5] Hidalgo-Mazzei D, Mateu A, Reinares M, Undurraga J, del Mar Bonnín C, Sánchez-Moreno J, Vieta E, Colom F, (2015) "Self-monitoring and psychoeducation in bipolar patients with a smart-phone application (SIMPLe) project: design, development and studies protocols," BMC Psychiatry, vol. 15, no. 52, pp. 1-9.

[6] Recio-Rodríguez JI, Martín-Cantera C, González-Viejo N, Gómez-Arranz A, Arietaleanizbeascoa MS, Schmolling-Guinovart Y, Maderuelo-Fernandez JA, Pérez-Arechaederra D, Rodriguez-Sanchez E, Gómez-Marcos MA, García-Ortiz L, on behalf the EVIDENT Group, (2014) "Effectiveness of a smartphones application for improving healthy lifestyles, a randomized clinical trial (EVIDENT II): study protocol," BMC Public Health, vol. 14, no. 254, pp. 1-13.

[7] Al-Hadithy N, Gikas PD, Al-Nammari SS, (2012) "Smartphones in orthopaedics. Int Orthop," vol. 2012, no. 36, pp. 1543-1547.

[8] Chittaro L, Sioni R, (2014) "Evaluating mobile apps for breathing training: the effectiveness of visualization," Comput Hum Behav, vol. 40, pp. 56-63.

[9] Saw AE, Main LC, Gastin PB, (2015) "Monitoring athletes through self-report: factors influencing implementation," J Sport Sci Med, vol. 14, no. 1, pp. 137-146.

[10] Conroy DE, Yang C-H, and Maher JP, (2014) "Behavior change techniques in top-ranked mobile apps for physical activity," Am J Prev Med, vol. 46, no. 6, pp. 649-652.

[11] 7 Minute Workout, 2016. 11. 3, Corporate homepage. http://www.7-min.com

[12] Daily Workout, 2016. 11. 3, Corporate homepage. http://www.dailyworkoutapps.com

[13] Sworkit, 2016. 11. 3, Corporate homepage. http://www.sworkit.com

[14] Parikh SV, Huniewicz P, (2014) "E-health: an overview of the uses of the Internet, social media, apps, and websites for mood disorders," Wolters Kluwer Health, vol. 28, no. 1, pp. 13-17.

[15] Endomondo, 2016. 11. 3, Corporate homepage. http://www.endomondo.com

[16] Nike Golf $360^{\circ}$ App, 2016. 11. 3, Corporate homepage. http://www.nike.com/us/en_us/c/golf/ng360

[17] Voice Caddie, 2016. 11. 3, Corporate homepage. http://www.voicecaddie.com

[18] 3baysGSA, 2016. 11. 3, Corporate homepage. http://www.3bayslife.com

[19] Smart Tennis Sensor, 2016. 11. 3, Corporate homepage. http://www.smarttennissensor.sony.net

[20] Budd L, Vorley T, (2013) "Airlines, apps, and business travel: a critical examination," Research in Transportation Business \& Management, vol. 9, pp. 41-49.

[21] ZEBRA, 2016. 11.3, http://www.zebra.com/gb/en/nfl.html

[22] NBA, 2016. 11. 3, http://stats.nba.com/tracking

[23] Lee K-H, Jeon Y-K, (2013) "Analysis of specific angle of joint on elite alpine skiers during slalom event," The Korea Journal of Sports Science, vol. 22, no. 5, pp. 1597-1608.

[24] Scheiber P, Seifert E, Muller E, (2012) "Relationships between biomechanics and physiology in older, recreational alpine skiers," Scand J Med Sci Spor, vol. 2012, no. 22, pp. 49-57.

[25] Sun M, Burke LE, Baranowski T, Fernstrom JD, Zhang H, Chen H-C, Bai Y, Li Y, Li C, Yue Y, Li Z, Nie J, Sclabassi RJ, Mao Z-H, Jia W, (2015) "An exploratory study on a chest-worn computer for evaluation of diet, physical activity and lifestyle," J Healthc Eng, vol. 6, no. 1, pp. 1-22.

[26] Zeal Optics Corporate homepage, 2016. 11. 3, http://www.zealoptics.com/technology/technologygoggles.html

[27] Banos O, Villalonga C, Damas M, Gloesekoetter P, Pomares H, Rojas I, (2014) "PhysioDroid: combining wearable health sensors and mobile devices for a ubiquitous, continuous, and personal monitoring," The Scientific World Journal, vol. 2014, no. 490824, pp. 1-11. 
International Journal of Software Engineering \& Applications (IJSEA), Vol.8, No.1, January 2017

[28] Boudreaux ED, Waring, ME, Hayes RB, Sadasivam RS, Mullen S, Pagoto S, (2014) "Evaluating and selecting mobile health apps: strategies for healthcare providers and healthcare organizations," TBM, vol. 2014, no. 4, pp. 363-371.

[29] Middelweerd A, Mollee JS, van der Wal CN, Brug J, Te Velde SJ, (2014) "Apps to promote physical activity among adults: a review and content analysis," Int J Behav Nutr Phy, vol. 11, pp. 97.

[30] Cranwell J, Benford S, Houghton RJ, Golembewksi M, Fischer JE, Hagger MS, (2014) "Increasing self-regulatory energy using an Internet-based training application delivered by smartphones technology," Cyberpsychology, Behavior, and Social Networking, vol. 17, no. 3, pp. 181-186.

[31] Glynn LG, Hayes PS, Casey M, Glynn F, Alvarez-Iglesias A, Newell J, ÓLaighin G, Heaney D, Murphy AW, (2013) "SMART MOVE - a smartphone-based intervention to promote physical activity in primary care: study protocol for a randomized controlled trial," Trials Journal, vol. 14, no. 157, pp. 1-7.

[32] Direito A, Jiang Y, Whittaker R, Maddison R, (2015) "Smartphones apps to improve fitness and increase physical activity among young people: protocol of the Apps for IMproving FITness (AIMFIT) randomized controlled trial,” BMC Public Health, vol. 15, no. 635, pp. 1-12.

\section{Authors}

Yong-Wook Kim He received the B.S., M.S in physics from Hanyang University, Korea in 1989, 1992. He received a degree of Sports science Ph.D. at Hanyang University in 2017. He has over 25 years of experience in developing computer software. Currently he is a senior researcher of the PSDC Sports science Research Institute. His main research field is the fusion of Sports science and computer software.

Sung-Rak Choi He was an elite baseball player back at his high school. He turned to golf because of the injury on his university. He passed KPGA and Q-school on 2012. He received a Ph.D. degree at Hanyang university on 2014. He is now an professor of Hanyang university teaching golf and run the research team working on sports industry.
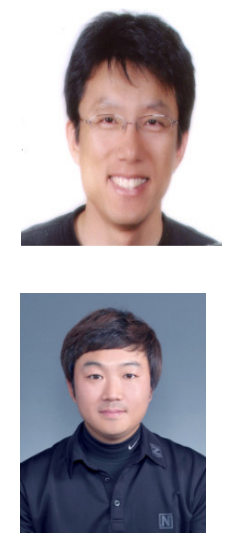